\title{
Evaluation on Fatigue Life of Expressway Asphalt Pavement Based on Tire-Pavement-Subgrade Coupling Model
}

\author{
Dong Cheng ${ }^{1,2,}$, Liu Wen-jie ${ }^{1,2}$, Zhou Lun ${ }^{1}$, Zhang Rui-lei ${ }^{1}$, Kan Qian-hua ${ }^{3}$, Leng Wu-ming ${ }^{2}$ \\ ${ }^{1}$ Hunan Communications Research Institute Limited Company, Changsha, China \\ ${ }^{2}$ School of Civil Engineering, Central South University, Changsha, China \\ ${ }^{3}$ School of Mechanics and Engineering, Southwest Jiaotong University, Chengdu, China
}

Email address:

dongcheng4321@126.com (Dong cheng), liuwje@163.com (Liu Wen-jie), 18769788330@163.com (Zhou Lun), 1454370514@qq.com (Zhang Rui-lei), qianhuakan@foxmail.com (Kan Qian-hua),1wm123456@126.com (Leng Wu-ming)

${ }^{*}$ Corresponding author

\section{To cite this article:}

Dong Cheng, Liu Wen-jie, Zhou Lun, Zhang Rui-lei, Kan Qian-hua, Leng Wu-ming. Evaluation on Fatigue Life of Expressway Asphalt Pavement Based on Tire-Pavement-Subgrade Coupling Model. American Journal of Civil Engineering. Vol. 5, No. 6, 2017, pp. $400-407$. doi: 10.11648/j.ajce.20170506.22

Received: November 14, 2017; Accepted: November 23, 2017; Published: December 28, 2017

\begin{abstract}
The fatigue failure has become one of the main failure modes in asphalt pavement of expressway. However, due to the material nonlinear of the tire, pavement and subgrade, the contact nonlinear of tire-pavement and the influence factors, such as the tire inflation pressure, rotation speed, wheel load, the values and distributions of contact stress between tire and pavement are extremely complex and affect the tensile stress and tensile strain, as well as affect the wear, deformation and fatigue life of pavement. The three dimensional (hereinafter referred to as 3D) finite element model of tire-subgrade-pavement structure was established, and the steady-stable rolling of tire and stress-dependent dynamic resilient modulus of subgrade soil were considered in the model. Based on the mixed Euler-Lagrange describtion, the steady-state finite element analysis were carried to indicate the mechanism of fatigue cracking of asphalt pavement, the influences of rolling resistance of wheel, friction coefficient, axle load, tire pressure and running velocity on fatigue lives were discussed based on the tensile strain fatigue prediction model. The results show that the fatigue life of pavement decreases with the increasing rolling resistance of wheel, friction coefficient, wheel load and tire pressure, and it does not obvisouly depend on the running velocity. The study could provide a reference for the design of the flexible asphalt pavement structure and the evaluation of pavement fatigue life.
\end{abstract}

Keywords: Asphalt Pavement, Finite Element Analysis, Dynamic Resilient Modulus, Steady-State Analysis, Fatigue Life

\section{Introduction}

On the damage of the expressway asphalt pavement, fatigue failure become one of the main form, and there are many influence factors among them, the study on fatigue damage of asphalt pavement always become hot spots and difficult points in the engineering and academia [1-3]. With the increase of traffic volume and axle weight, the damage to the road surface is becoming more and more serious. Under the repeated action of the vehicle load, the road is in the stress and strain state of the long tension alternation, the strength of the roadbed and pavement structure decreased.
When the load accumulates to a certain amount, the internal force produced by the load exceeds the surface structure resistance, the pavement structure will crack, and the strength will decrease rapidly until fatigue failure occurs. According to statistical analysis, special section of the highway, such as the band, the top and bottom of the long longitudinal slopes, the joint between the end of the long longitudinal slope (the bottom of the slope) and the small radius of the bend, and the bridge and tunnel transition section, and so on, fatigue cracks are more likely to occur because of the high shear force and horizontal friction [4].

In the study of fatigue damage on asphalt pavement, many domestic scholars have conducted a large number of 
researches. Based on the theory of fracture mechanics, Qiu yang-yang, Chang'an University [5], the prediction method of fatigue life of asphalt pavement based on local strain was proposed, the strain rule of asphalt concrete pavement was simulated through finite element method, and the reliability of the theory was verified through the combination of asphalt mixture strain fatigue equation and Miner linear fatigue cumulative damage theory. Yang bo [6] put forward the track estimate method and calculation process of bituminous pavement based on the finite element model, and proposed the suggestion of pavement maintenance and design method of thickness according to the development trend of pavement. The reliability of the method was verified by comparison with the practical example. WANG Bao-liang [7] think it is inappropriate to use the maximum tensile stress of the asphalt base as fatigue parameters of asphalt pavement structure in our country, the fatigue damage factor should be expressed by the stress amplitude with the maximum tensile stress and compressive stress. LIU Jie [8] got some useful conclusions by studying the interaction between the real tyres and the steep slopes. Howevre, the load was mostly reduced to a circle or rectangle in the previous study, it was difficult to obtain the true response of the asphalt pavement in the direct contact part of the wheel. The tire is an indispensable part of the vehicle-roadbed coupling analysis model, reasonable tire finite element model is the key of the whole analysi. Meanwhile, fatigue life is related to the steady rolling contact of the wheel - road surface, static analysis results will result in large deviations $[9,10]$. With the improvement of finite element technology and computer performance, the finite element software is used to analyze the complex engineering structures, it is possible to establish a more complete finite element model of tire-subgrade-pavement structure to design and analyze the highway.

In order to obtain more accurate stress response of asphalt pavement, a 3D finite element model of tire-subgrade-pavement structure was established in this paper based on a steady-state analysis method of the mixed Euler-Lagrange describtion provided by ABAQUS [10], and the steady-stable rolling of tire and stress-dependent dynamic resilient modulus of subgrade soil were considered in the model, the mechanism of fatigue cracking of asphalt pavement was revealed, the influences of rolling resistance of wheel, friction coefficient, axle load, tire pressure and running velocity on fatigue lives were discussed based on the tensile strain fatigue prediction equation of the asphalt mixture. The study could provide a reference for the design of the flexible asphalt pavement structure and the evaluation of pavement fatigue life.

\section{The 3D Finite Element Model of Tire-Subgrade-Pavement Structure Coupling Analysis}

The method of ABAQUS (a finite element software) steady-state rolling analysis was adopted for tire-subgrade-pavement structure coupling analysis. In this analysis, euler algorithm was applied to tire rolling, and lagrange algorithm was applied to pavement deformation calculation, Thus, the steady state rolling process can be achieved with limited grid number. Steady-state rolling analysis requires the streamline to be set in the tire model, the streamline is automatically generated by rotating the two-dimensional axisymmetric model around the axis to generate a 3D model, as shown in Figure 1. The constitutive model and parameters of tire material are selected according to the paper of Feng lin-ge [11], the standard inflation pressure of $11 \mathrm{R} 20$ tire is $930 \mathrm{kPa}$, the load capacity of a single tire in the double wheel load is $32.5 \mathrm{kN}$, and the single wheel is $35.5 \mathrm{kN}$. The three-dimensional model is obtained by rotation, and the results are shown in figure 2 .

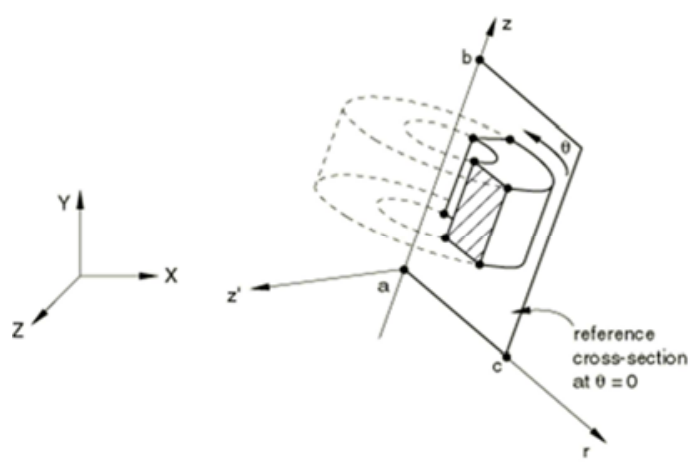

Figure 1. Sketch diagram of the $3 D$ model generation.

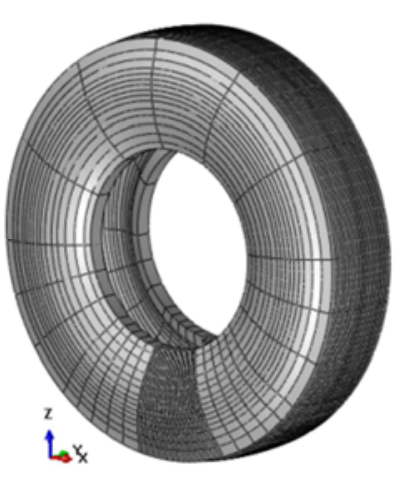

Figure 2. 3D model of the tire.

The horizontal dimension of asphalt pavement structure was 2.5 to $2.5 \mathrm{~m}$, and the vertical dimension was $4.76 \mathrm{~m}$. For load trucks, a complete single axle contains four tires, in order to reduce the calculation, only the unilateral wheel axisymmetric modeling was taken. In the case of uniaxial double-wheel, the structure was symmetric about the center of the wheel gap, therefore, it was possible to establish a finite element analysis of only $1 / 4$ of the original model. The research focuses on the response of the pavement part of asphalt pavement under the action of vehicle load, therefore, a fine mesh was divided into the direct contact between the road and the tire, the rest of the grid was in a gradient sparse grid, symmetric boundary conditions were applied to the symmetric surface, as shown in Figure 3. The 3D tire-subgrade-pavement finite element model 
was built by assembling the tire model and the roadbed model and setting the tire and pavement surface - surface contact, as shown in Figure 4.

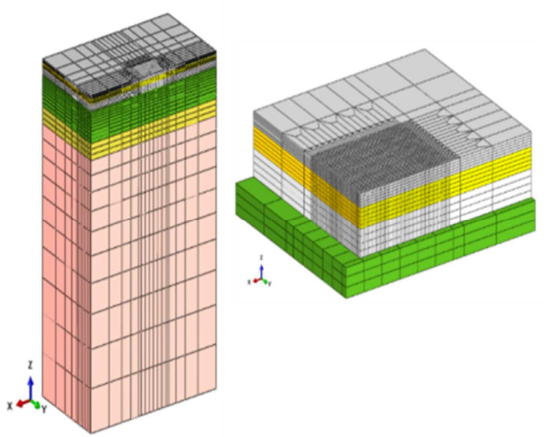

Figure 3. The whole grid of subgrade and the local refined grid.

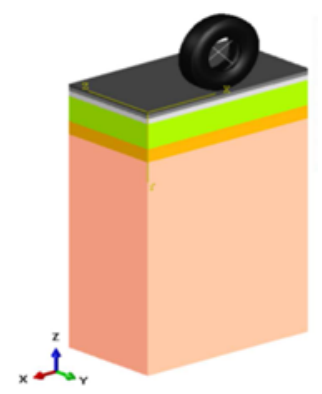

Figure 4. Tire- subgrade-pavement 3D coupling model.

At present, the design of pavement structure in China is based on the elastic laminar system[12-13], the static elastic modulus of subgrade was used to characterize its overall mechanical properties, and the stress state correlation was not considered. However, in the actual pavement structure, the stress state of subgrade was spatial distribution function, therefore, the modulus of the subgrade also changed with the space position. In order to obtain more accurate stress-strain response of roadbed pavement, the modulus of roadbed soil was adopted by the dynamic modulus model of highway subgrade (The N37A model for short) presented by the America research report of NCHRP1-37A [14], the model equation was as follows:

$$
M_{r}=k_{1} P_{a}\left(\frac{\theta}{P_{a}}\right)^{k_{2}}\left(\frac{\tau_{o c t}}{P_{a}}+1\right)^{k_{3}}
$$

In formula (1), $M_{\mathrm{r}}$ is the modulus of resilience, $\theta$ is the body stress $\tau_{\text {oct }}$ is the octahedral shear stress, $k_{1}, k_{2}$ and $k_{3}$ is the regression coefficient of the stress correlation of subgrade soil, $p_{a}$ is atmospheric pressure, which is $100 \mathrm{kPa}$. In order to achieve dynamically based on the state of stress related rebound modulus of subgrade pavement structure analysis, the uniform tangent stiffness matrix of N37A is derived based on the user material subprogram UMAT provided by ABAQUS, and based on the generalized linear elastic hooke's law. Similar to linear elastic constitutive relations, the constitutive relation of stress-related elastic materials can be written as follows:

$$
\mathrm{S}=\frac{M_{r}}{1+v}(\alpha \varepsilon \mathrm{I}+\mathrm{E})
$$

Where $\mathrm{E}$ is the strain tensor, $\varepsilon$ is the body strain, The expression is $\varepsilon=\operatorname{tr}(\mathrm{E}), \alpha=v /(1-2 v)$ Make $\mathrm{C}(\theta, \tau$ oct $)$ to:

$$
C\left(\theta, \tau_{o c t}\right)=\frac{M_{r}\left(\theta, \tau_{o c t}\right)}{1+v}=k P_{a}\left(\frac{\theta}{P_{a}}\right)^{k_{1}}\left(\frac{\tau_{o c t}}{P_{a}}+1\right)^{k_{2}}
$$

Where $k=k_{1} /(1+v)$. In combination with (2) and (3), stress related elastic constitutive relation can be simplified as:

$$
\mathrm{S}=C\left(\theta, \tau_{\text {oct }}\right)(\alpha \varepsilon \mathrm{I}+\mathrm{E})
$$

Finally, the consistent tangent stiffness matrix of the formula (4) is derived, as shown in equation (5):

$$
\frac{\partial \mathrm{S}}{\partial \mathrm{E}}=C\left(1+d_{1} \mathrm{I} \otimes \mathrm{I}+d_{2} \overline{\mathrm{S}} \otimes \mathrm{I}+d_{3} \mathrm{I} \otimes \overline{\mathrm{S}}+d_{4} \overline{\mathrm{S}} \otimes \overline{\mathrm{S}}\right)
$$

Where $\quad d_{1}=\alpha+m \frac{\bar{\alpha} k_{2}\left(\tau_{o c t}+P_{a}\right)}{3}, \quad d_{2}=m \frac{\bar{\alpha} k_{2}\left(\tau_{o c t}+P_{a}\right)}{\sigma}$, $d_{3}=m \frac{\sigma k_{3}}{9 \tau_{o c t}}, d_{4}=m \frac{k_{3}}{3 \tau_{\text {oct }}}$

The process of related derivation and ABAQUS finite element transplantation can be used to refer to previous work [15]. The material parameters of roadbed pavement are shown in table 1 , the dynamic resilience modulus model of roadbed

\begin{tabular}{|c|c|c|c|c|c|}
\hline \multirow{2}{*}{\multicolumn{2}{|c|}{ The name of the structure layer }} & \multicolumn{4}{|c|}{ Parameters of the structure layer } \\
\hline & & Modulus of resilience / MPa & density/ $\left({\left.\mathrm{g} . \mathrm{cm}^{-3}\right)}^{-3}\right.$ & Poisson's ratio & thickness / cm \\
\hline \multicolumn{2}{|c|}{ SMA layer } & 1800 & 2.50 & 0.35 & 4 \\
\hline \multicolumn{2}{|c|}{$\mathrm{AC}-20$ layer } & 1600 & 2.50 & 0.35 & 6 \\
\hline \multicolumn{2}{|c|}{ AC-25 layer } & 1500 & 2.50 & 0.35 & 8 \\
\hline \multicolumn{2}{|c|}{ Cement gravel layer (5\%) } & 3200 & 2.40 & 0.20 & 38 \\
\hline \multicolumn{2}{|c|}{ Cement gravel layer (4\%) } & 3200 & 2.30 & 0.20 & 20 \\
\hline \multirow{3}{*}{ subgrade } & 96 district & N37A & 1.87 & 0.35 & 80 \\
\hline & 93 district & N37A & 1.87 & 0.35 & 70 \\
\hline & 90 district & N37A & 1.87 & 0.35 & 100 \\
\hline \multicolumn{2}{|l|}{ base } & 20 & 1.30 & 0.40 & 150 \\
\hline
\end{tabular}
soil adopts the model parameters of standard water content, cement content of $4 \%$, compactness of $90 \%, 93 \%$ and $96 \%$ [16].

Table 1. The material parameters of the subgrade-pavement. 


\section{Stress and Strain Response Characteristics of Asphalt Pavement Under Tire Load}

The different contact state of tire and road surface has great influence on the stress of road surface, in the special section of highway, the vehicle is often in constant braking and acceleration, and the road surface is more prone to wear and tear. To study the effect of different contact state of pavement stress, the pavement stress response of these four conditions of static load, free rolling, fully acceleration slip and fully brake slip are analyzed under the service conditions of wheel load $32.5 \mathrm{kN}$, tire pressure $930 \mathrm{kpa}$, the friction coefficient 0.6 , the result is shown in figure 5 . The analysis showed that when the vehicle was in static load and free rolling state, the maximum principal stress of the road was mainly compressive stress, and the tensile stress value is small, and it is not easy to form cracks. When the vehicle is in a state of accelerated slippage or brake slip completely, the tensile stress zone of the road surface maximum principal stress increases, formed the crescent-shaped tensile stress area, and the highway road surface in the process of serving the observed u-shaped cracks phenomenon, a crescent tensile stress region is formed, which is consistent with the u-shaped cracks observed in the road surface during the course of service.

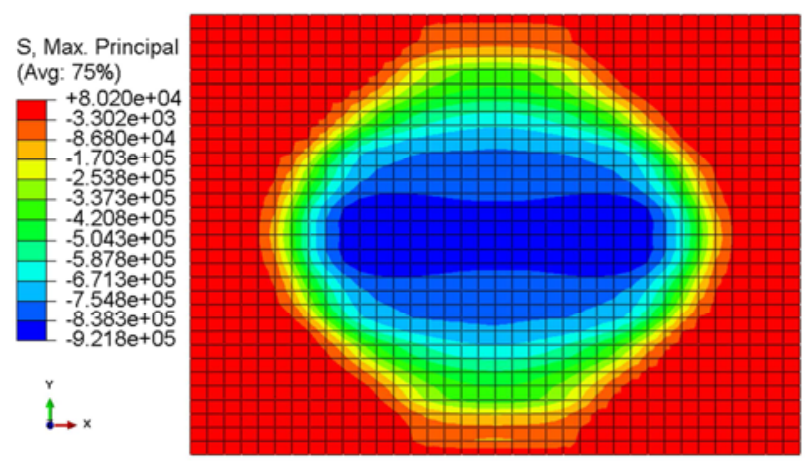

(a) Static load

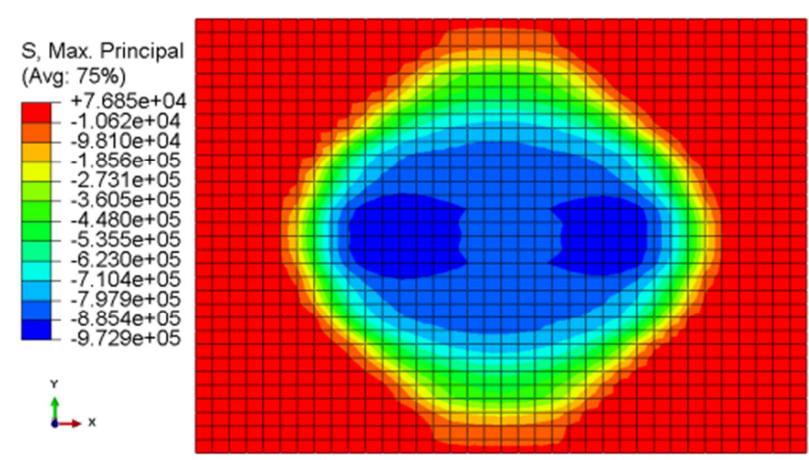

(b) Free rolling

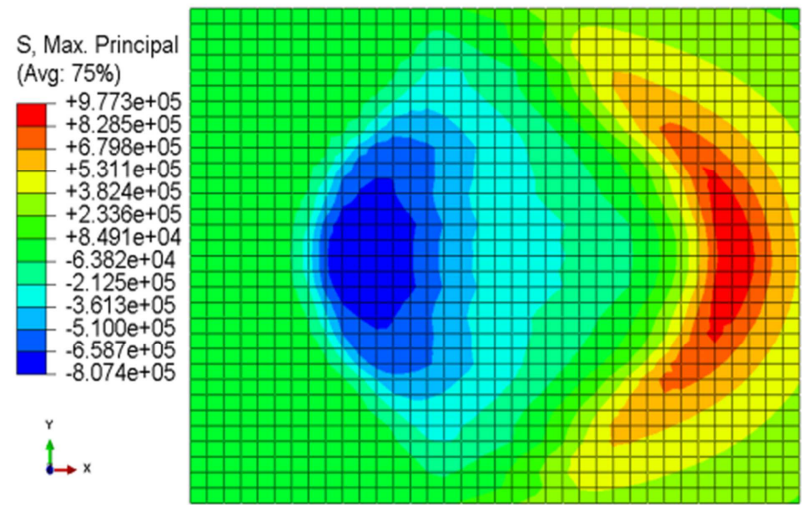

(c) Fully acceleration slip

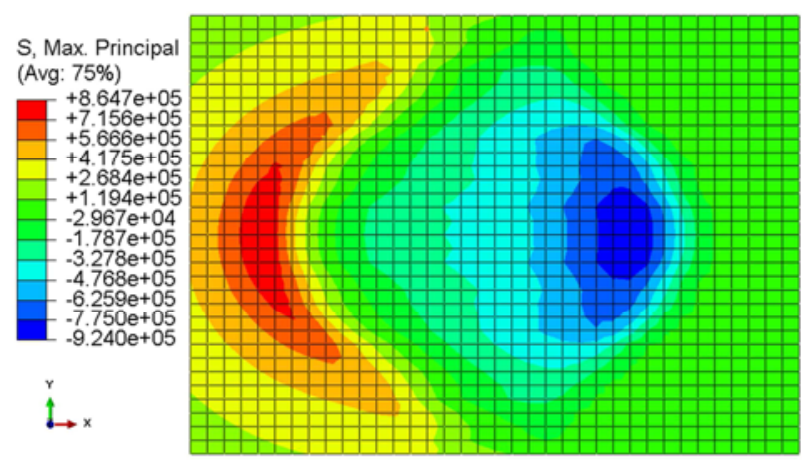

(d) Fully brake slip

Figure 5. The maximum principal stress distribution of pavement under different driving conditions.

Figure 6 shows the maximum principal stress curve of asphalt layer and bottom layer along the pavement. When the tire is free to roll, under the action of centrifugal force, the contact area of the tire is smaller than the static load state, and the stress area is more concentrated and tensile stress is greater. For full acceleration slip and full braking slip, the stress region of peak value is formed at the reverse of acceleration. In addition, due to the large stiffness of the asphalt pavement structure of semi-rigid base asphalt pavement, the tensile stress of the asphalt layer under repeated wheel loading is very small.

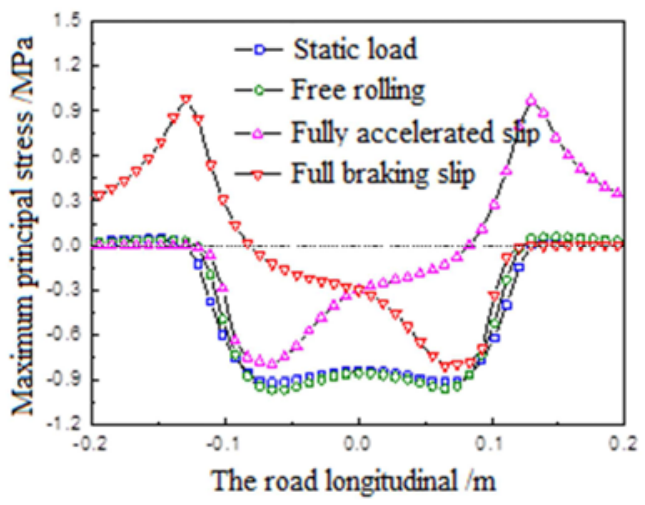

(a) Asphalt surface course 


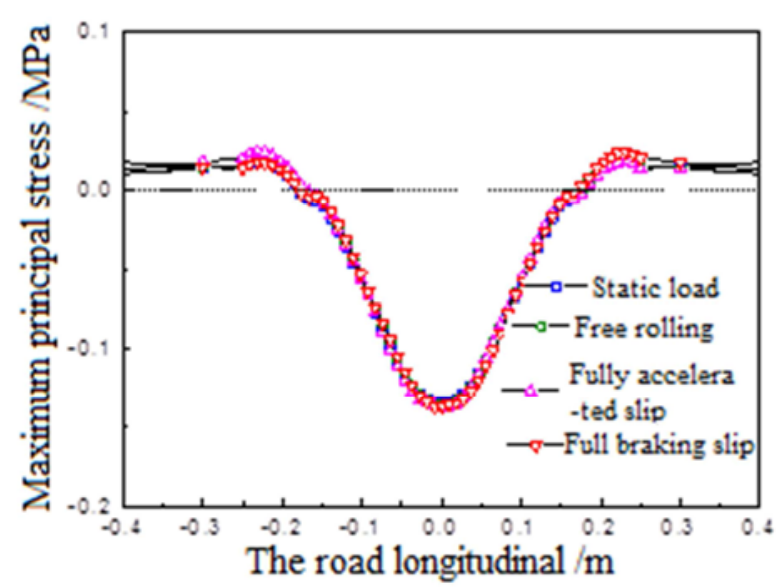

(b) The bottom of the asphalt layer
The stress diagram of the road surface under vehicle driving condition is shown in Figure 7, it can be seen that the surface of the road is always subjected to the pulpation cycle, which is the important cause of the u-shaped crack. In this paper, the horizontal counterforce of the wheel shaft is $0.155 \mathrm{kN}, 6.374 \mathrm{kN}, 12.151 \mathrm{kN}$ and $19.415 \mathrm{kN}$, respectively corresponding to the four driving conditions mentioned above. As shown in Figure 8, when the horizontal force is $0.16 \mathrm{kN}$, the horizontal reaction is small, which is equivalent to the free rolling state of the tire. The base asphalt of the tire is in total pressure and strain state, once the tire is slippage, there will be a high tensile stress belt of the crescent shape on the face layer. As the slide of the tire increases, the slip belt gradually moves to the front of the tire as the sliding level increases, while the compressive zone spreads to the rear, finally, a peak point of tensile stress appears in the front of the tire - road contact.

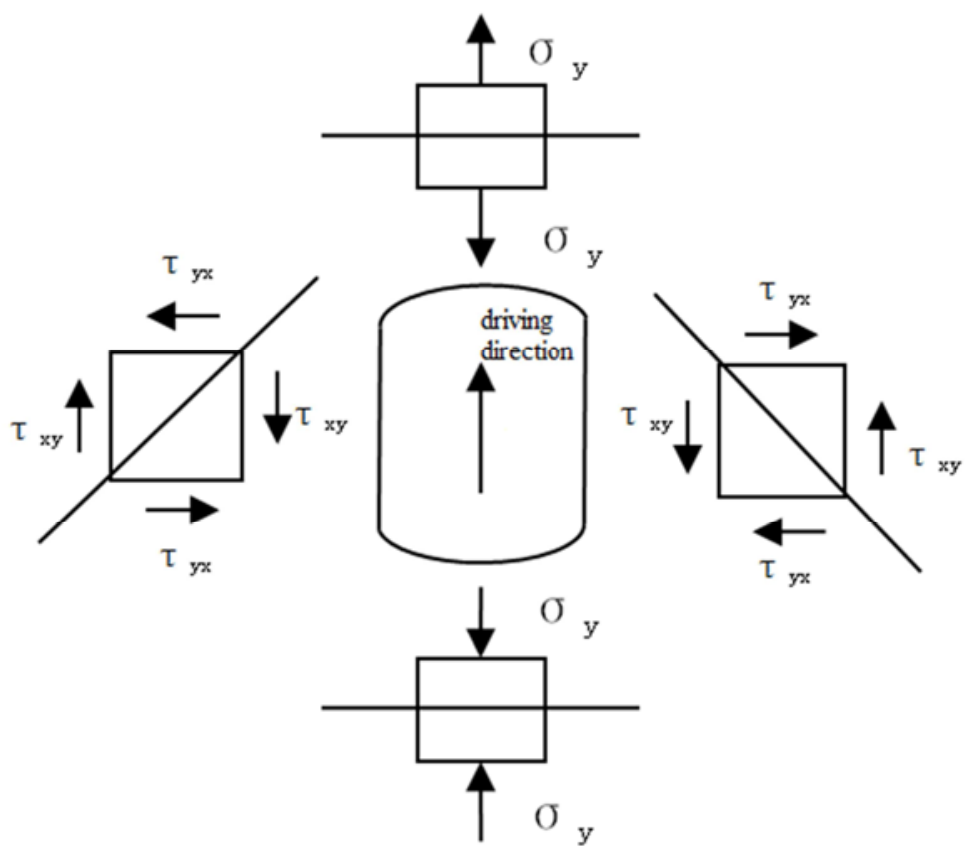

Figure 7. Sketch diagram of the pavement stress.

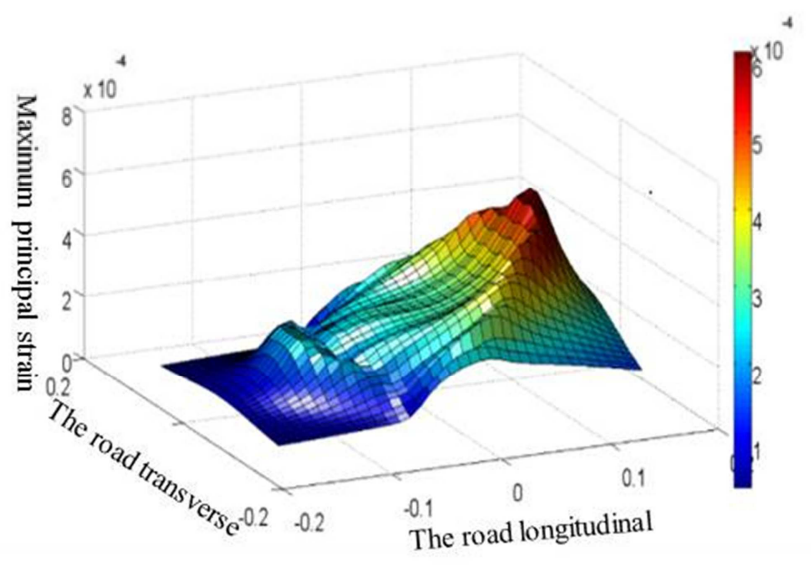

Figure 8. The main strain of the pavement.

\section{Prediction Analysis of Fatigue Life of Asphalt Pavement}

\subsection{Prediction Model of Fatigue Life of Asphalt Pavement}

The asphalt surface is made of bituminous horseshoe gravel mixed material (SMA), which is a bituminous mixture that is filled with bitumen in coarse aggregate skeleton, which has excellent anti-rutting and anti-slip performance. The type and content of fiber in filling material have important influence on SMA. Wang hui [17] analyzed the influence of fiber on the anti-rutting performance, creep performance, high temperature stability and fatigue life of SMA, as shown in Table 2. The life prediction model of SMA types can be obtained by regression analysis of strain and fatigue life. 
Table 2. Test results and regression equation of fatigue life for different fiber SMA.

\begin{tabular}{lllll}
\hline Fiber category & micro strain $\left(\mathbf{1 0}^{-6}\right)$ & life span (One million time) & equation of prediction & correlation coefficient \\
\hline \multirow{2}{*}{ Wood fiber } & 200 & 33.7 & $N_{f}=2.15325 \times 10^{12} \varepsilon^{-4.701}$ & $\mathrm{R}^{2}=0.9988$ \\
& 300 & 4.57 & & \\
polyacrylonitrile & 400 & 1.31 & $N_{f}=1.9988 \times 10^{11} \varepsilon^{-4.6722}$ & $\mathrm{R}^{2}=0.9683$ \\
& 200 & 18.34 & & \\
mineral fibre & 300 & 3.50 & $N_{f}=3.2187 \times 10^{12} \varepsilon^{-4.6722}$ & $\mathrm{R}^{2}=0.9997$ \\
& 200 & 57.83 & & \\
\hline
\end{tabular}

Note: $N_{f}$-Fatigue life frequency (One million time), $\varepsilon$-micro strain.

\subsection{Prediction of Fatigue Life of Asphalt Pavement}

In combination with the fatigue life prediction model of the mineral fiber asphalt horseshoe gravel mixed materials in table 2, the influence curve of the shaft resistance shown in Figure 9 on the maximum principal strain and fatigue life of the pavement is presented. Figure 9 shows that the axial resistance is basically linear relationship with the main pull of the road surface, and the life of the road decreases sharply with the increase of tensile strain; When the shaft resistance is $0.155 \mathrm{kN}$, the maximum main strain of the road is $66 \mathrm{e}-6$, and the number of road fatigue can reach 9.56e 7 times; When the wheel is completely slippage, the shaft resistance is $19.415 \mathrm{kN}$, the strain is $553 \mathrm{e}-6$, and the life expectancy is reduced to 4500 , which is 21000 times compared with the free rolling. Therefore, it is easier to damage the road surface in the upper and lower slopes, the small radius curves and the transition sections of the bridge where rapid braking are easier to occur.

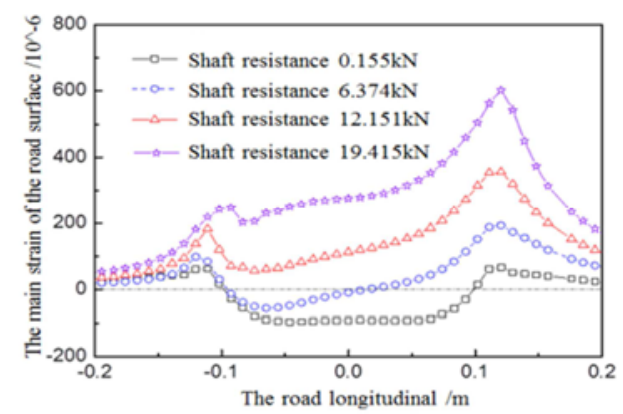

(a) Road main strain

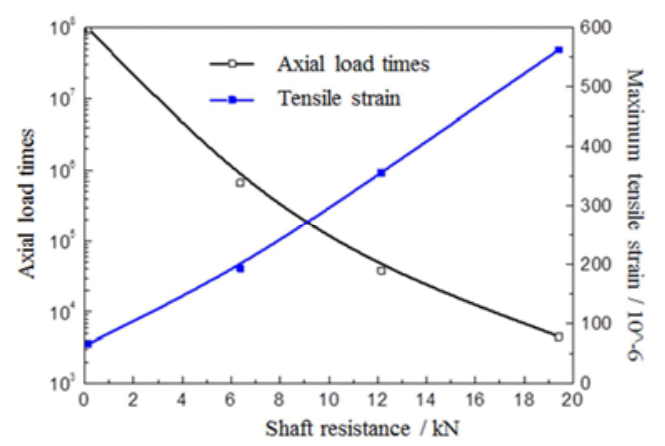

(b) The road life

Figure 9. The relationship curve of the shaft resistance between maximum principal strain and fatigue life.
The rolling state of the tire directly affects the service life of the road, and the friction coefficient of the road, the speed of the tire, the wheel load and the tire pressure are directly related to the rolling of the tire. Figure 10 shows the influence of the friction coefficient on the maximum principal stress and fatigue life of the road under the initial free rolling state (the speed of the tire is equal to the speed of the tire). The analysis shows that, with the increase of friction coefficient, the peak of maximum principal strain increases rapidly, resulting in that the fatigue life reduced from $7.75 \mathrm{e} 6$ to $2.35 \mathrm{e} 6$.

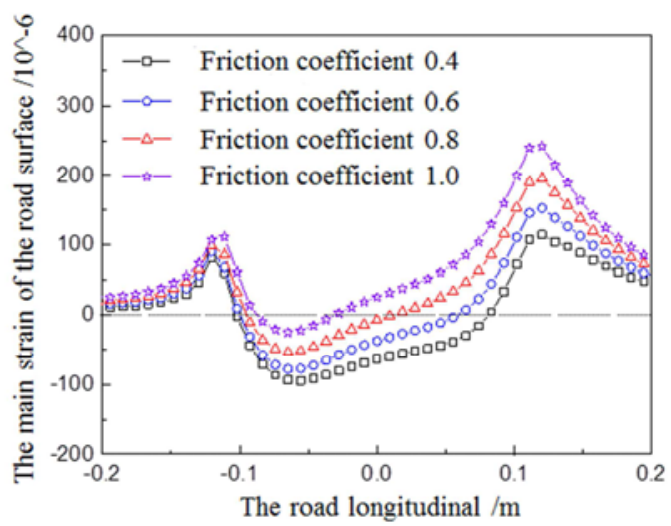

(a) Road main strain

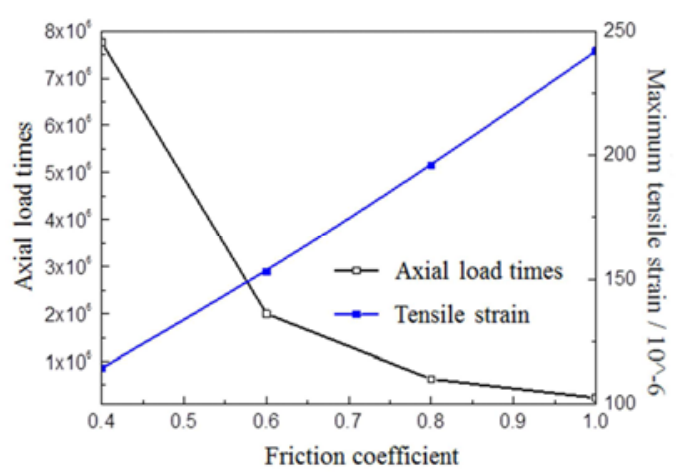

(b) The road life

Figure 10. The influence of friction coefficient on the maximum principal strain and fatigue life of pavement.

Figure 11 shows the effect of vehicle speed on the maximum principal strain of the road under the initial free rolling state, it can be seen that the maximum principal strain 
of pavement has little relation to the speed of operation.

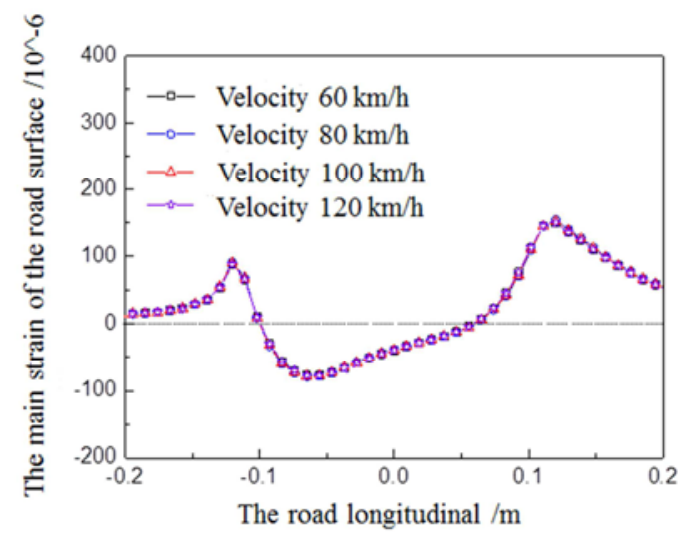

(a) Road main strain

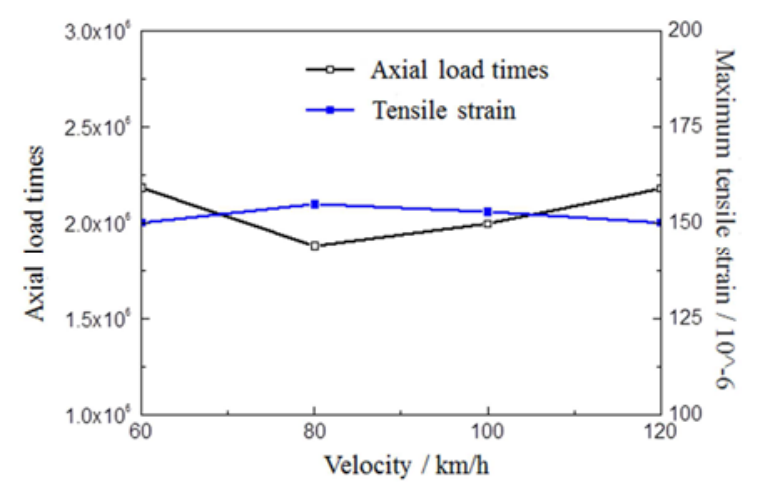

(b) The road life

Figure 11. The influence of speed on maximum principal strain and pavement fatigue life.

Figure 12 shows the influence of the wheel load on the maximum principal strain and fatigue life under the initial free rolling state. With the increase of wheel load, the maximum principal strain and wheel load do not have a monotonically changing relationship, thus the effect on life span is also the same. In general, the effect of wheel load on pavement fatigue life is less obvious than that of friction coefficient. When the wheel load increased from $27 \mathrm{kN}$ to $35.5 \mathrm{kN}$, the fatigue life was reduced from $2.88 \mathrm{e} 6$ to $1.42 \mathrm{e} 6$, it's almost doubled.

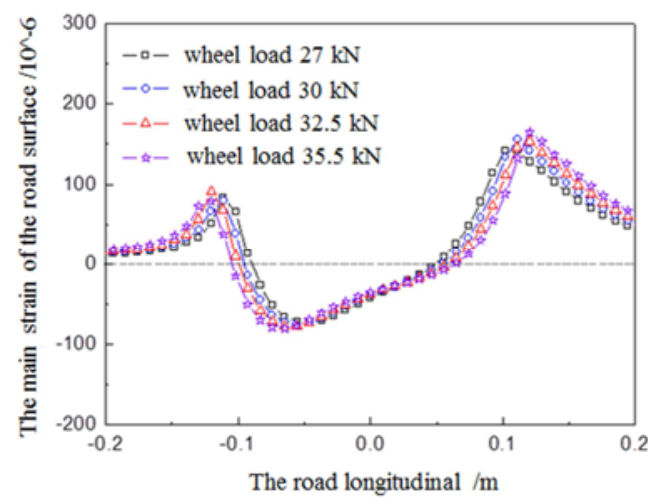

(a) Road main strain

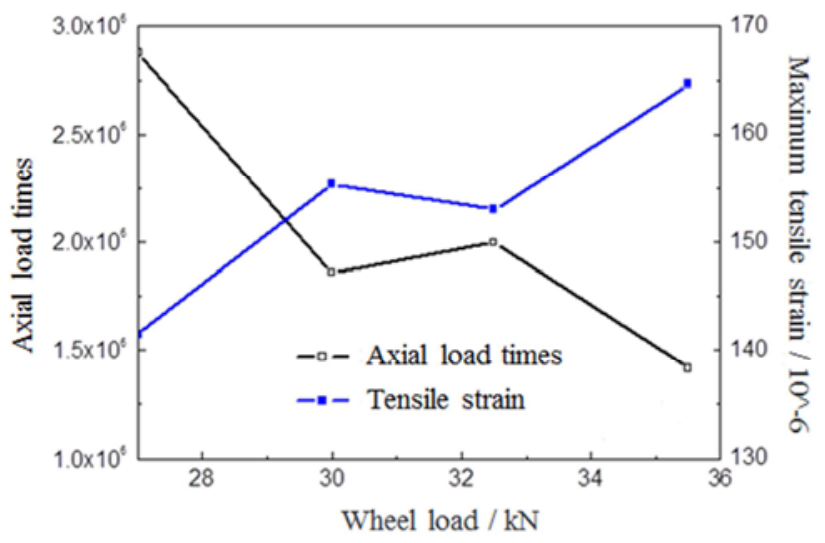

(b) The road life

Figure 12. The influence of wheel load on maximum principal strain and pavement fatigue life.

Figure 13 shows the influence of tire pressure on the maximum principal strain and fatigue life of the pavement under the initial free rolling state. It can be seen that as the tire pressure increases, the maximum principal strain decreases and the fatigue life increases correspondingly. When the tire pressure increased from $800 \mathrm{kPa}$ to $1000 \mathrm{kPa}$, the fatigue life decreased from $2.47 \mathrm{e} 6$ to $1.63 \mathrm{e} 6$.

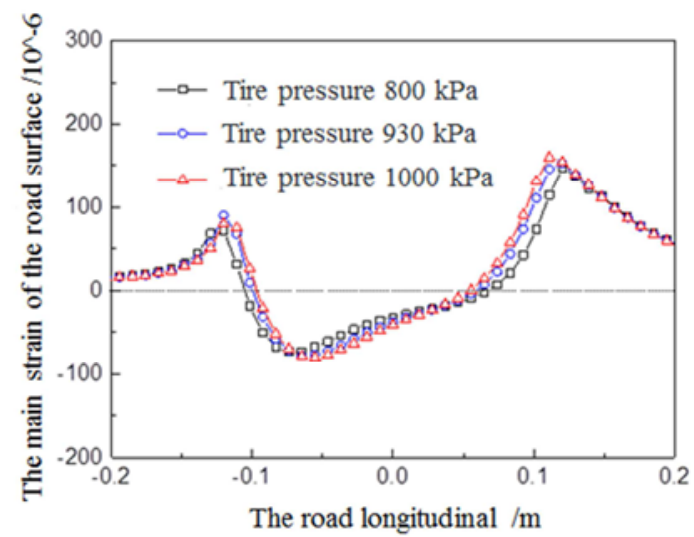

(a) Road main strain

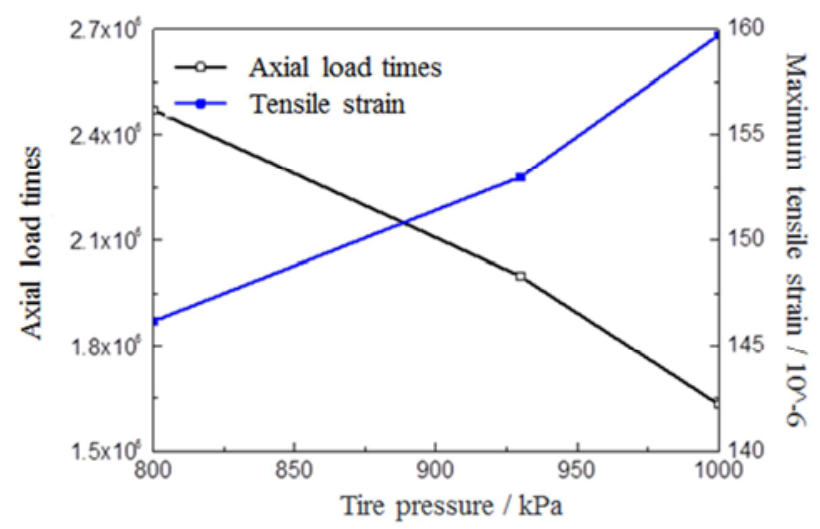

(b) The road life

Figure 13. The influence of tire pressure on maximum principal strain and pavement fatigue life. 
It can be seen from the above discussion that the influence of different factors on pavement fatigue life surface is different in the initial free rolling state, the importance of influencing factors is the rotation resistance, friction coefficient, wheel load, tire pressure and running speed.

\section{Conclusion}

In order to obtain a more accurate stress-strain response of asphalt pavement, a three-dimensional finite element model of the tire - pavement - roadbed structure was established, and the stress correlation of the modulus of resilience of the roadbed was considered, the steady-state rolling analysis of tire - pavement - roadbed was carried out based on the mixed Euler-Lagrange describtion.

In combination with the influence of tire acceleration and deceleration on the road stress, tensile strain and shear stress, the formation mechanism of $U$ type crack was revealed, the effect of the shaft resistance on the fatigue life of the road was obtained. The results showed that with the increase of the shaft resistance, the high tensile stress and strain zone appeared in the front of the tire, and the fatigue load of the road decreased sharply, the load number of the road surface was only 4,500 when the tire was fully skidding, which was one in two parts per million of the free roll.

The results of finite element analysis in the initial free rolling state showed that the fatigue life of pavement increases with the increase of shaft resistance, friction coefficient, wheel load and tire pressure, and the shaft resistance and friction coefficient are the most obvious.

\section{Acknowledgements}

This work was supported by the Youth Talents Support Program of Hunan (grant number 2016RS3037); Fund of Post-doctoral Studies. The authors would like to thank all the members of Key Laboratory at Hunan Communication Research Institute, Changsha, China.

\section{References}

[1] Tian bo, Zhao dui-jia. Stress analysis of cement concrete pavement with heavy loading [J]. China Journal of Highway \& Transport, 2000, 02:16-19.

[2] Gong xiu-qing. Research on Macrotexture Wear and Skid Resistance Degradation of Asphalt Pavement [D]. Beijing university of technology, 2014.
[3] Yang jun, Li wei-nong, Cheng zhi-wei, et al. Finite element analysis of asphalt pavement on long-steep longitudinal slope [J]. Journal of Traffic \& Transportation Engineering, 2010:20-24.

[4] Xu zhi-hong, Li shu-ming, Gao ying, et al. Research on Fatigue Characteristic of Asphalt Mixture [J]. Journal of Traffic \& Transportation Engineering, 2001.01:20-24.

[5] Qiu yang-yang. Study on Local strain and fatigue life Estimation based on elastic-plastic finite element method for asphalt pavement [D]. Chang'an University, 2013.

[6] Yang bo. Influencing Factors of Asphalt Pavement Rutting and Applications Based on Finite Element Method [D]. Chang'an University, 2010.

[7] Wang bao-liang. The study of fatigue behavior of asphalt pavement under vehicle load [D], Chang' an University, 2008.

[8] Liu jie. The finite element study of the interaction between the tyre and the steep incline [D]. Chongqing Jiaotong University, 2012.

[9] Xie rui-li, Kan qian-hua, Kang guo-zheng, et al. Plastic Deformation Analysis for Different Kinds of High Strength Rail Steels Under Stable Wheel-Rail Contact [J]. Applied Mathematics \& Mechanics, 2014. S1:127-130.

[10] Mei zuo-zhou. Research on fatigue damage of highway asphalt pavement based on mixed lagrange-euler algorithm [D]. Southwest Jiaotong University, 2014.

[11] Feng lin-ge. Finite Element Analysis of 11.00R20 All Steel Radial Truck Tire [D]. Qingdao University of Science and Technology, 2012.

[12] JTG D40-2002. Specifications of cement concrete pavement design for highway $[\mathrm{S}]$.

[13] JTG D50-2006. Specifications for design of highway asphalt pavement [S].

[14] Cheng sheng-kai, Ling jian-ming, Luo zhi-gang. Stress-dependent characteristics and prediction model of the resilient modulus of subgrade soils [J]. China Civil Engineering Journal, 2007, 40(6): 95-99.

[15] Ning xia-yuan, Dong cheng, Li zhi-yong, et al. A FEM-based method to implement the model of dynamic resilient modulus [J]. Rock and Soil Mechanics, 2015, 36(4): 1182-1188.

[16] Dong cheng, Leng wu-ming, Li zhi-yong, et al. Experimental study of dynamic resilient modulus of the cement improved high liquid limit clay [J]. Rock and Soil Mechanics, 2013, 34(1): 133-138.

[17] Wang hui. Study on different Fibres Influences on Pavement Performances of SMA [D]. Changsha University of Science \& Technology, 2007. 